\title{
ON THE NEWTON-LIKE METHOD FOR THE INCLUSION OF A POLYNOMIAL ZERO*
}

\author{
Ljiljana D. Petković, Miodrag S. Petković
}

\begin{abstract}
One of the most important problems in solving nonlinear equations is the construction of such initial conditions which provide both the guaranteed and fast convergence of the considered numerical algorithms. In this paper we study an iterative method of NEWTON's type for the inclusion of isolated complex zero of a given polynomial. We state computationally verifiable initial condition for the convergence of the considered interval method, which depends only on attainable data, and prove the quadratic convergence of this method. A numerical example is given.
\end{abstract}

\section{INTRODUCTION}

During the last forty years various techniques for a posteriori error estimates for the approximation of polynomial complex zeros were developed, see [6]. A quite different and efficient approach to error estimates uses complex circular arithmetic, as pointed out by GARGANTINI and HENRICI [3]. Iterative methods realized in circular interval arithmetic produce resulting disks that contain complex zeros of a polynomial. In this manner, not only very close approximations to the zeros (given by the centers of disks) but also the upper error bounds for the zeros (given by the radii of disks) are provided. More details about interval methods for the inclusion of polynomial zeros can be found in $[\mathbf{1}],[\mathbf{6}],[\mathbf{7}],[\mathbf{1 0}]$ and references cited there.

The aim of this paper is to present an iterative method of NEWTON's type for the inclusion of an isolated complex zero of a polynomial. A special attention will be paid to the construction of computationally verifiable initial condition that provides the guaranteed convergence of the proposed method. Let us stress that this subject, known in literature as a composite part of "point estimation theory"

${ }^{*}$ This research was supported by the Serbian Ministry of Science under grant number 144024G 2000 Mathematics Subject Classification. 65H05, 65G20, 30C15.

Key Words and Phrases. Interval method, polynomial zeros, inclusion of zeros, convergence, initial conditions., 
(SMALE [11]), is one of the most important problems in the theory and practice of iterative processes which attracts a great attention in recent time (for more details see $[8])$.

To state the interval method for the inclusion of polynomial complex zeros, we need the basic properties of circular interval arithmetic. A disk $Z$ with center $\operatorname{mid} Z=c$ and radius $\operatorname{rad} Z=r$, that is $Z:=\{z:|z-c| \leq r\}$, will be denoted briefly by the parametric notation $Z=\{c ; r\}$. The set of all complex circular intervals (disks) is denoted by $K(\mathbb{C})$. The basic circular arithmetic operations are defined as follows:

$$
\begin{aligned}
& \alpha\{c ; r\}=\{\alpha c ;|\alpha| r\} \quad(\alpha \in \mathbb{C}) \\
& \left\{c_{1} ; r_{1}\right\} \pm\left\{c_{2} ; r_{2}\right\}=\left\{c_{1} \pm c_{2} ; r_{1}+r_{2}\right\} \\
& \left\{c_{1} ; r_{1}\right\} \cdot\left\{c_{2} ; r_{2}\right\}=\left\{c_{1} c_{2} ;\left|c_{1}\right| r_{2}+\left|c_{2}\right| r_{1}+r_{1} r_{2}\right\} .
\end{aligned}
$$

The inversion of a disk $Z=\{c ; r\}$ which does not contain the origin (that is, $|c|>r$ holds) is defined by the MöBIUs transformation,

$$
Z^{-1}=\left\{\frac{1}{z}: z \in\{c ; r\}\right\}=\left\{\frac{\bar{c}}{|c|^{2}-r^{2}} ; \frac{r}{|c|^{2}-r^{2}}\right\} .
$$

Following the introduced inversion, division is defined as

$$
Z_{1}: Z_{2}=Z_{1} \cdot Z_{2}^{-1} \quad\left(0 \notin Z_{2}\right) \text {. }
$$

For two disks $Z_{1}=\left\{c_{1} ; r_{1}\right\}$ and $Z_{2}=\left\{c_{2} ; r_{2}\right\}$ the following is valid:

$$
\begin{aligned}
& \left\{c_{1} ; r_{1}\right\} \cap\left\{c_{2} ; r_{2}\right\}=\emptyset \quad \Leftrightarrow \quad\left|c_{1}-c_{2}\right|>r_{1}+r_{2}, \\
& \left|c_{1}-c_{2}\right| \leq r_{1}-r_{2} \quad \Leftrightarrow \quad\left\{c_{1} ; r_{1}\right\} \subseteq\left\{c_{2} ; r_{2}\right\} .
\end{aligned}
$$

A fundamental property of interval computation is the inclusion isotonicity which forms the basis for almost all applications of interval arithmetic. Let $f$ be a complex function over a given disk $Z \in K(\mathbb{C})$. The complex-valued set $\{f(z) \mid z \in$ $Z\}$ is not a disk in general. To deal with disks, we introduce an circular extension $F$ of $f$, defined on a subset $D \subseteq K(\mathbb{C})$ such that

$$
\begin{aligned}
& F(Z) \supseteq\{f(z) \mid z \in Z\} \quad \text { for all } Z \in D \quad \text { (inclusion), } \\
& F(z)=f(z) \quad \text { for all } z \in Z \quad \text { (complex restriction). }
\end{aligned}
$$

We shall say that the complex interval extension $F$ is inclusion isotone if the implication

$$
Z_{1} \subseteq Z_{2} \Rightarrow F\left(Z_{1}\right) \subseteq F\left(Z_{2}\right)
$$

is satisfied for all $Z_{1}, Z_{2} \in D$. In particular, we have

$$
z \in Z \Rightarrow f(z)=F(z) \in F(Z) \text {. }
$$

Let us note that the four basic operations in circular complex arithmetic are inclusion isotone ([1, Ch. 5]). 


\section{NEWTON-LIKE INTERVAL METHOD}

Let

$$
P(z)=z^{n}+a_{n-1} z^{n-1}+\cdots+a_{1} z+a_{0}=\prod_{j=1}^{n}\left(z-\zeta_{j}\right) \quad\left(a_{i} \in \mathbb{C}\right)
$$

be a monic polynomial of degree $n$ with simple complex zeros $\zeta_{1}, \ldots, \zeta_{n}$. Let us assume that we have found a $\operatorname{disk} A:=\{z:|z-a| \leq \eta\}$, shortly denoted by parametric notation $\{a ; \eta\}(a=\operatorname{mid} A, \eta=\operatorname{rad} A)$, that contains only one zero of $P$. All other zeros are supposed to lie in the region $W=\{w:|w-a|>\eta\}=\operatorname{ext} A$, that is, in the exterior of the disk $A=\{a ; \eta\}$ (see Fig. 1). There is a lot of results for the localization of polynomial zeros (see, e.g., MARDEN [5], Henrici [4]) and we will not discuss this subject in this paper. We also note that a useful computational test for the existence of polynomial zero can be found in [9]. Without loss of generality, we will adopt that the sought zero is denoted by $\zeta_{1}$; moreover, we write $\zeta$ instead of $\zeta_{1}$. In our study we always assume that $n \geq 3$.

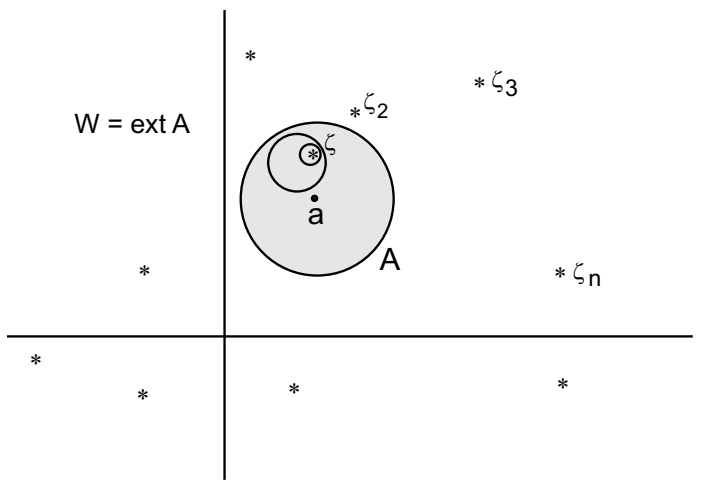

Figure 1.

Using the logarithmic derivative, we find

$$
\frac{P^{\prime}(z)}{P(z)}=\sum_{j=1}^{n} \frac{1}{z-\zeta_{j}}=\frac{1}{z-\zeta}+\sum_{j=2}^{n} \frac{1}{z-\zeta_{j}}
$$

We single out the zero $\zeta_{1}=\zeta$ from (5) and obtain the fixed point relation

$$
\zeta=z-\frac{1}{\frac{P^{\prime}(z)}{P(z)}-\sum_{j=2}^{n}\left(z-\zeta_{j}\right)^{-1}} .
$$

According to the inclusion isotonicity property (4) we have $\left(z-\zeta_{j}\right)^{-1} \in$ $(z-W)^{-1}$ for any $z \in\{a ; \eta\}$ and $j=2,3, \ldots, n$. Since $z \notin W$, that is, $|z-a|<\eta$, 
the inversion of the open region $z-W$ is a closed interior of a circle given by

$$
H=(z-W)^{-1}=\left\{w:\left|w+\frac{\bar{z}-\bar{a}}{\eta^{2}-|z-a|^{2}}\right| \leq \frac{\eta}{\eta^{2}-|z-a|^{2}}\right\}=\{h ; d\},
$$

where

$$
h=\operatorname{mid} H=\frac{\bar{a}-\bar{z}}{\eta^{2}-|z-a|^{2}}, \quad d=\frac{\eta}{\eta^{2}-|z-a|^{2}} .
$$

(see Gargantini [2]). Taking into account that $\zeta_{j} \in W$, we return to (6) and obtain

$$
\zeta \in z-\frac{1}{\frac{P^{\prime}(z)}{P(z)}-\sum_{j=2}^{n}(z-W)^{-1}}=z-\frac{1}{\frac{P^{\prime}(z)}{P(z)}-(n-1)\{h ; d\}}
$$

The relation (7) suggests the construction of an iterative method for the inclusion of isolated zero of the polynomial $P$. Let $Z^{(m)}=\left\{z^{(m)} ; r^{(m)}\right\}$ be a disk with center $z^{(m)}=\operatorname{mid} Z^{(m)}$ and radius $r^{(m)}=\operatorname{rad} Z^{(m)}(m=0,1, \ldots)$. For an initial inclusion disk we take $Z^{(0)}=\{a ; \eta\}=A$, that is, $z^{(0)}=a$ and $r^{(0)}=\eta$. We will use the following abbreviations:

$$
H^{(m)}=\left\{h^{(m)} ; d^{(m)}\right\}, \quad h^{(m)}=\frac{\bar{a}-\bar{z}^{(m)}}{\eta^{2}-\left|z^{(m)}-a\right|^{2}}, \quad d^{(m)}=\frac{\eta}{\eta^{2}-\left|z^{(m)}-a\right|^{2}} .
$$

Starting from (7) we can construct the following iterative method for the inclusion of one zero of the given polynomial $P$ :

$$
Z^{(m+1)}=z^{(m)}-\frac{1}{\frac{P^{\prime}\left(z^{(m)}\right)}{P\left(z^{(m)}\right)}-(n-1)\left\{h^{(m)} ; d^{(m)}\right\}} \quad(m=0,1, \ldots),
$$

where $z^{(m)}=\operatorname{mid} Z^{(m)}$.

REMARK 1. The iterative formula (8), written in the form,

$$
Z^{(m+1)}=z^{(m)}-\frac{P\left(z^{(m)}\right)}{P^{\prime}\left(z^{(m)}\right)} \cdot \frac{1}{1-\frac{P\left(z^{(m)}\right)}{P^{\prime}\left(z^{(m)}\right)}(n-1)\left\{h^{(m)} ; d^{(m)}\right\}} \quad(m=0,1, \ldots),
$$

obviously resembles the NEWTON method. For this reason, we will refer (8) to as the NEWTON-like interval method.

REMARK 2. Regarding the role of the disk $H^{(m)}$ in (8), we can conclude that it does not have any influence to the convergence rate of the iterative method (8). However, the main role of the interval $H^{(m)}=\left\{h^{(m)} ; d^{(m)}\right\}$ is to provide the inclusion of the zero $\zeta$ within the disk $Z^{(m+1)}$. 
Remark 3. The interval method (8) is a modification of the Gargantini-Henrici method $[\mathbf{3}]$ of the third order

$$
Z_{i}^{(m+1)}=z_{i}^{(m)}-\frac{1}{\frac{P^{\prime}\left(z_{i}^{(m)}\right)}{P^{\prime}\left(z_{i}^{(m)}\right)}-\sum_{\substack{j=1 \\ j \neq i}}^{n}\left(z_{i}^{(m)}-Z_{j}^{(m)}\right)^{-1}} \quad(i=1, \ldots, n ; m=0,1, \ldots) .
$$

for the simultaneous inclusion of all simple zeros of the polynomial $P$.

\section{CONVERGENCE ANALYSIS}

In this section we will study the convergence behavior of the NEWTON-like method (8). Our main goal is to state computationally verifiable initial condition that enables the guaranteed convergence of this method. As a consequence, we prove that the NEWTON-like method (8) has the quadratic convergence.

Assume that we have found an initial disk $Z^{(0)}=\{a ; \eta\}$ that contains one and only one zero $\zeta$ of $P$ and let the inequality

$$
\left|\frac{P(a)}{P^{\prime}(a)}\right|<\frac{\eta}{3(n-1)}
$$

hold. We note that this inequality involves only known data: the center and radius of the initial inclusion disk $Z^{(0)}=A=\{a ; \eta\}$ and the polynomial degree $n$. We will show later that, if the inequality (9) is valid, then the iterative method (8) is convergent with the quadratic convergence. Let us stress that the initial condition of the form (9) is of great practical importance since it depends only on attainable data.

Let us introduce the quantity

$$
\rho^{(m)}=\eta-\left|z^{(m)}-a\right| \quad(m=0,1, \ldots) .
$$

Lemma 1. The following implication is valid:

$$
\left|\frac{P(a)}{P^{\prime}(a)}\right|<\frac{\eta}{3(n-1)} \Rightarrow \rho^{(1)}>5(n-1) r^{(1)} .
$$

Proof. The inversion of the open region $T=\{z:|z-t|>R\}$, when $0 \notin T$ (that is, $|t|<R)$, is given by

$$
T^{-1}=\left\{\frac{-\bar{t}}{R^{2}-|q|^{2}} ; \frac{R}{R^{2}-|q|^{2}}\right\} .
$$

By using (10) we obtain

$$
\frac{n-1}{z^{(0)}-W}=(n-1)\left\{0 ; \frac{1}{\eta}\right\}=\left\{0 ; \frac{n-1}{\eta}\right\} \quad\left(z^{(0)}=a\right) .
$$


The iterative formula (8) for $m=0$ has a simple form

$$
Z^{(1)}=a-\frac{1}{\left\{\frac{P^{\prime}(a)}{P(a)} ; \frac{n-1}{\eta}\right\}}
$$

Having in mind the inequality (9) we find

$$
\left|\frac{P^{\prime}\left(z^{(0)}\right)}{P\left(z^{(0)}\right)}\right|=\left|\frac{P^{\prime}(a)}{P(a)}\right|>\frac{3(n-1)}{\eta}>\frac{n-1}{\eta}=\operatorname{rad} \frac{n-1}{z^{(0)}-W} .
$$

Therefore, according to (2), the disk in the denominator of (8) does not contain 0 when $m=1$, which means that $Z^{(1)}$ is also a disk.

We apply (1) and (9) and estimate

$$
\begin{aligned}
r^{(1)} & =\operatorname{rad} Z^{(1)}=\operatorname{rad} \frac{1}{\left\{\frac{P^{\prime}(a)}{P(a)} ; \frac{n-1}{\eta}\right\}}=\frac{\frac{n-1}{\eta}}{\left|\frac{P^{\prime}(a)}{P(a)}\right|^{2}-\frac{(n-1)^{2}}{\eta^{2}}} \\
& <\frac{\frac{n-1}{\eta}}{\left[\frac{3(n-1)}{\eta}\right]^{2}-\frac{(n-1)^{2}}{\eta^{2}}}=\frac{\eta}{8(n-1)} \leq \frac{\eta}{16} .
\end{aligned}
$$

In the similar way, using (9) we find

$$
\begin{aligned}
\left|z^{(1)}-z^{(0)}\right| & =\left|z^{(1)}-a\right|=\frac{\left|\frac{P^{\prime}(a)}{P(a)}\right|}{\left|\frac{P^{\prime}(a)}{P(a)}\right|^{2}-\frac{(n-1)^{2}}{\eta^{2}}} \\
& <\frac{\frac{3(n-1)}{\eta}}{\left[\frac{3(n-1)}{\eta}\right]^{2}-\frac{(n-1)^{2}}{\eta^{2}}}=\frac{3 \eta}{8(n-1)}
\end{aligned}
$$

Using (11) and (12) we obtain

$$
\rho^{(1)}=\eta-\left|z^{(1)}-a\right|>\eta-\frac{3 \eta}{8(n-1)}=\frac{8 n-11}{8(n-1)} \eta \geq \frac{13}{16} \eta>5(n-1) r^{(1)} .
$$

In this way we have proved that the initial condition (9) implies the inequality

$$
\rho^{(1)}>5(n-1) r^{(1)},
$$

which has the important role in the convergence analysis. 
Let us note that $\zeta \in Z^{(1)}$ according to the inclusion isotonicity property. We will now consider the iterative method (8) for $m \geq 1$ starting with the inclusion disk $Z^{(1)}$ and the condition (14). This condition holds if (9) is valid, which will not be further particularly cited. Using circular arithmetic operations, the iterative formula (8) can be rewritten in the form

$$
Z^{(m+1)}=z^{(m)}-\left\{c^{(m)} ; d^{(m)}\right\}^{-1} \quad(m=0,1, \ldots),
$$

where

$$
c^{(m)}=\frac{P^{\prime}\left(z^{(m)}\right)}{P\left(z^{(m)}\right)}+\frac{(n-1)\left(\bar{z}^{(m)}-\bar{a}\right)}{\eta^{2}-\left|z^{(m)}-a\right|^{2}}, \quad d^{(m)}=\frac{(n-1) \eta}{\eta^{2}-\left|z^{(m)}-a\right|^{2}} .
$$

For simplicity, in what follows we will omit the iteration index always when there is no possibility of a confusion.

Lemma 2. If the inequality

$$
\rho>5(n-1) r
$$

is valid, then $0 \notin\{c ; d\}$ and

$$
\frac{|c|}{|c|^{2}-d^{2}}<\frac{15}{8} r
$$

Proof. Using (5) and (16) we find for $m \geq 2$

$$
\begin{aligned}
|c| & >\frac{1}{|z-\zeta|}-\sum_{j=2}^{n} \frac{1}{\left|z-\zeta_{j}\right|}-\frac{(n-1)|z-a|}{\eta^{2}-|z-a|^{2}} \\
& >\frac{1}{r}-\frac{n-1}{\rho}-\frac{(n-1)(\eta-\rho)}{\eta^{2}-(\eta-\rho)^{2}}>\frac{3}{5 r}
\end{aligned}
$$

and

$$
d=\frac{(n-1) \eta}{\eta^{2}-|z-a|}=\frac{(n-1) \eta}{\eta^{2}-(\eta-\rho)^{2}}<\frac{n-1}{\rho} .
$$

Since

$$
|c|>\frac{3}{5 r}>\frac{3}{5} \cdot \frac{5(n-1)}{\rho}>\frac{n-1}{\rho}>d,
$$

according to (2) it follows that $0 \notin\{c ; d\}$.

To prove the second assertion of the lemma, we use (16), (18) and (19) to find

$$
\frac{|c|}{|c|^{2}-d^{2}}<\frac{\frac{3}{5 r}}{\left(\frac{3}{5 r}\right)^{2}-\frac{(n-1)^{2}}{\rho^{2}}}<\frac{\frac{3}{5 r}}{\left(\frac{3}{5 r}\right)^{2}-\frac{1}{5 r^{2}}}<\frac{15}{8} r .
$$


Theorem 1. Let the sequence of disks $\left\{Z^{(m)}\right\}$ be defined by the iterative method (8), assuming that the initial disk $Z^{(0)}=\{a ; \eta\}$ is chosen so that the condition (9) is satisfied. Then, the Newton-like method (8) is convergent, and the following is true in each iterative step:

$$
\begin{aligned}
& 1^{\circ} \quad \zeta \in Z^{(m)} \\
& 2^{\circ} \quad r^{(m+1)}<\frac{25(n-1)}{4 \eta}\left[r^{(m)}\right]^{2} .
\end{aligned}
$$

Proof. The proof of the assertion $1^{\circ}$ follows from the construction of the method (8), based on the inclusion isotonicity and the relation $(7)$, and the fact that $z^{(m)} \in$ $\{a ; \eta\}$ for each $m=0,1, \ldots$, which is obvious because of $\eta-\left|z^{(m)}-a\right|=\rho^{(m)}>$ $5(n-1) r^{(m)}>0$.

We recall that the initial condition (9) implies the inequality (14), which will be used in the convergence analysis. From (15) we obtain

$$
r^{(2)}=\operatorname{rad} Z^{(2)}=\frac{d^{(1)}}{\left|c^{(1)}\right|^{2}-\left[d^{(1)}\right]^{2}} .
$$

Hence, using (14), (18) and (19), we find

$$
\begin{aligned}
r^{(2)} & <\frac{n-1}{\rho^{(1)}\left[\left(\frac{3}{5 r^{(1)}}\right)^{2}-\left(\frac{n-1}{\rho^{(1)}}\right)^{2}\right]}<\frac{n-1}{\rho^{(1)}\left[\left(\frac{3}{5 r^{(1)}}\right)^{2}-\left(\frac{1}{5 r^{(1)}}\right)^{2}\right]} \\
& <\frac{25(n-1)\left[r^{(1)}\right]^{2}}{8 \rho^{(1)}} .
\end{aligned}
$$

Starting from (20), we find by (14)

$$
r^{(2)}<\frac{25(n-1) r^{(1)}}{8 \rho^{(1)} / r^{(1)}}<\frac{5}{8} r^{(1)}
$$

which means that the disk $Z^{(2)}$ is contracted compared to $Z^{(1)}$. Using Lemma 2 we obtain

$$
\rho^{(2)}>\eta-\left|z^{(2)}-a\right|=\eta-\left|z^{(1)}-a-\frac{\bar{c}^{(1)}}{\left|c^{(1)}\right|^{2}-\left[d^{(1)}\right]^{2}}\right|>\rho^{(1)}-\frac{\left|\bar{c}^{(1)}\right|}{\left|c^{(1)}\right|^{2}-\left[d^{(1)}\right]^{2}},
$$

that is,

$$
\rho^{(2)}>\rho^{(1)}-\frac{15}{8} r^{(1)}
$$

Taking into account (14), (21) and (22), we find

$$
\rho^{(2)}>\rho^{(1)}-\frac{15}{8} r^{(1)}>5(n-1) r^{(1)}-\frac{15}{8} r^{(1)}>\frac{8}{5} r^{(2)}\left[5(n-1)-\frac{15}{8}\right],
$$


and whence, for $n \geq 3$,

$$
\rho^{(2)}>5(n-1) r^{(2)} .
$$

Therefore, we have proved the implications

$$
\left|\frac{P(a)}{P^{\prime}(a)}\right|<\frac{\eta}{3(n-1)} \Rightarrow \rho^{(1)}>5(n-1) r^{(1)} \Rightarrow \rho^{(2)}>5(n-1) r^{(2)} .
$$

This chain of implications has the key role in the proof by induction. Besides, since (23) holds, then it follows $0 \notin\left\{c^{(2)} ; d^{(2)}\right\}$ and the inclusion method (15) is well defined for $m=2$.

Assume that for $m \geq 2$ the following is true:

$$
\begin{aligned}
r^{(m)} & <\frac{25(n-1)\left[r^{(m-1)}\right]^{2}}{8 \rho^{(m-1)}}, \\
r^{(m)} & <\frac{5}{8} r^{(m-1)} \\
\rho^{(m)} & >5(n-1) r^{(m)} \\
\rho^{(m)} & >\rho^{(m-1)}-\frac{8}{5} r^{(m-1)} .
\end{aligned}
$$

These inequalities have already been proved for $m=2$. We will prove that they are valid for the index $m+1$.

Applying the above consideration for $m=2$ and (26), we obtain

$$
r^{(m+1)}<\frac{25(n-1)\left[r^{(m)}\right]^{2}}{8 \rho^{(m)}}<\frac{5}{8} r^{(m)} .
$$

In the similar way as for $m=1$, it is easy to show that

$$
\rho^{(m+1)}>5(n-1) r^{(m+1)} \quad \text { and } \quad \rho^{(m+1)}>\rho^{(m)}-\frac{8}{5} r^{(m)} .
$$

By the successive application of (25) and (27), we find

$$
\begin{aligned}
\rho^{(m)} & >\rho^{(m-1)}-\frac{8}{5} r^{(m-1)}>\rho^{(m-2)}-\frac{8}{5} r^{(m-2)}-\frac{8}{5} r^{(m-1)} \\
& >\rho^{(m-2)}-\frac{8}{5} r^{(m-2)}-\frac{8}{5} \cdot \frac{5}{8} r^{(m-2)}=\rho^{(m-2)}-\frac{8}{5} r^{(m-2)}\left(1+\frac{5}{8}\right) \\
& \vdots \\
& >\rho^{(1)}-\frac{8}{5} r^{(1)}\left(1+\frac{5}{8}+\left(\frac{5}{8}\right)^{2}+\cdots\right)>\rho^{(1)}-\frac{8}{5} \cdot \frac{1}{1-\frac{5}{8}} r^{(1)}>\rho^{(1)}-5 r^{(1)} .
\end{aligned}
$$

Using (11) and (13) we estimate

$$
\rho^{(m)}>\rho^{(1)}-5 r^{(1)}>\frac{13}{16} \eta-5 \cdot \frac{\eta}{16}=\frac{\eta}{2} .
$$


Substituting this bound in (28) we obtain

$$
r^{(m+1)}<\frac{25(n-1)\left[r^{(m)}\right]^{2}}{8 \rho^{(m)}}<\frac{25(n-1)\left[r^{(m)}\right]^{2}}{8(\eta / 2)}=\frac{25(n-1)}{4 \eta}\left[r^{(m)}\right]^{2}
$$

and the assertion $2^{\circ}$ is proved. The last relations point to the quadratic convergence of the NewTon-like method (8).

The interval method (15) is well defined in each iterative step since $0 \notin$ $\left\{c^{(m)} ; d^{(m)}\right\}$ (according to Lemma 2). The convergence of the NEWTON-like method (8) follows according to (25); indeed, the sequence of radii $\left\{r^{(m)}\right\}$ converges to 0.

Finally, let us note that $Z^{(m)} \subset A=\{a ; \eta\}$ for each $m$, thus, there is no possibility that any disk $Z^{(m)}$ includes zeros lying outside the initial disk $A$ (see Fig. 1). Indeed, since $\rho^{(m)}>5(n-1) r^{(m)}>r^{(m)}$, we have

$$
\rho^{(m)}=\eta-\left|z^{(m)}-a\right|>r^{(m)}, \quad \text { that is, } \quad\left|z^{(m)}-a\right|<\eta-r^{(m)} .
$$

Hence, according to (3), it follows $Z^{(m)}=\left\{z^{(m)} ; r^{(m)}\right\} \subset\{a ; \eta\}=A$.

\section{NUMERICAL EXAMPLE}

We have applied the NEWTON-like method (8) to the algebraic polynomial

$$
P(z)=z^{7}+z^{5}-10 z^{4}-z^{3}-z+10
$$

with the zeros $2, \pm 1, \pm i,-1 \pm 2 i$. Applying methods for the existence of zeros and their localization, and the proximity test for detecting zeros, we have found that the disk $A=\{0.1+0.9 i ; 1.5\}$ contains only one zero of $P$. Inclusion disks obtained by (8) are given below:

$$
\begin{aligned}
& Z^{(1)}=\{0.14083+1.07444 i ; 0.1976\}, \\
& Z^{(2)}=\{0.010327+0.974089 i ; 0.08369\}, \\
& Z^{(3)}=\{-0.001419+1.001066 i ; 0.00344\}, \\
& Z^{(4)}=\left\{-4.88 \times 10^{-6}+0.99999699 i ; 1.27 \times 10^{-5}\right\}, \\
& Z^{(5)}=\left\{5.88 \times 10^{-11}+0.999999999987 i ; 1.32 \times 10^{-10}\right\}, \\
& Z^{(6)}=\left\{-1.04 \times 10^{-21}+0.9999999999999999999934 i ; 1.46 \times 10^{-20}\right\} .
\end{aligned}
$$

All presented disks contain the exact zero $\zeta=i$. 


\section{REFERENCES}

1. G. Alefeld, J. Herzberger: Introduction to Interval Computation. Academic Press, New York 1983.

2. I. GARGANTINI: Further application of circular arithmetic: Schröder-like algorithms with error bound for finding zeros of polynomials. SIAM J. Numer. Anal., 15 (1978), 497-510.

3. I. Gargantini, P. Henrici: Circular arithmetic and the determination of polynomial zeros. Numer. Math., 18 (1972), 305-320.

4. P. Henrici: Applied and Computational Complex Analysis, Vol. I. John Wiley and Sons, New York 1974.

5. M. Marden: The Geometry of Polynomials. Mathematical surveys. Amer. Math. Soc., Providence, Rhode Island 1966.

6. M. S. Petković: Iterative Methods for Simultaneous Inclusion of Polynomial Zeros. Springer-Verlag, Berlin-Heidelberg-New York 1989.

7. M. S. PetKović: On the Halley-like algorithms for the simultaneous approximation of polynomial complex zeros. SIAM J. Numer. Anal., 26 (1989), 740-763.

8. M. S. Petković, Đ. D. Herceg, S. M. Ilić: Point Estimation Theory and its Applications. Institute of Mathematics, Novi Sad 1997.

9. M. S. Petković, Lj. D. Petković: On a computational test for the existence of polynomial zero. Comput. Math. with Appls., 17 (1989), 1109-1114.

10. M. S. Petković, LJ. D. Petković:, Complex Interval Arithmetic and its Applications. Wiley-VCH, Berlin-Weinhein-New York 1998.

11. S. Smale: The fundamental theorem of algebra and complexity theory. Bull. Amer. Math. Soc., 4 (1981), 1-35.

Ljiljana D. Petković,

(Received September 11, 2006)

Faculty of Mechanical Engineering,

University of Niš, 18000 Niš,

Serbia

E-mail: Ijiljana@masfak.ni.ac.yu

Miodrag S. Petković,

Faculty of Electronic Engineering,

University of Niš, $18000 \mathrm{Niš,}$

Serbia

E-mail: msp@eunet.yu 